\title{
nuckle invilade...
}

\section{LA LEY DE SALUD MENTAL COLOMBIANA EN EL CONTEXTO LATIONOAMERICANOz UNA APUESTA POR EL RESPETO A LOS DERECHOS HUMANOS}

\author{
Jesús Andrés Sánchez S. ${ }^{\dagger+}$, Laura Carolina Castro B. ${ }^{\ddagger \neq \neq}$, \\ John Pedraza P.§§§, Mariam Verbel R. ${ }^{* * * *}$
}

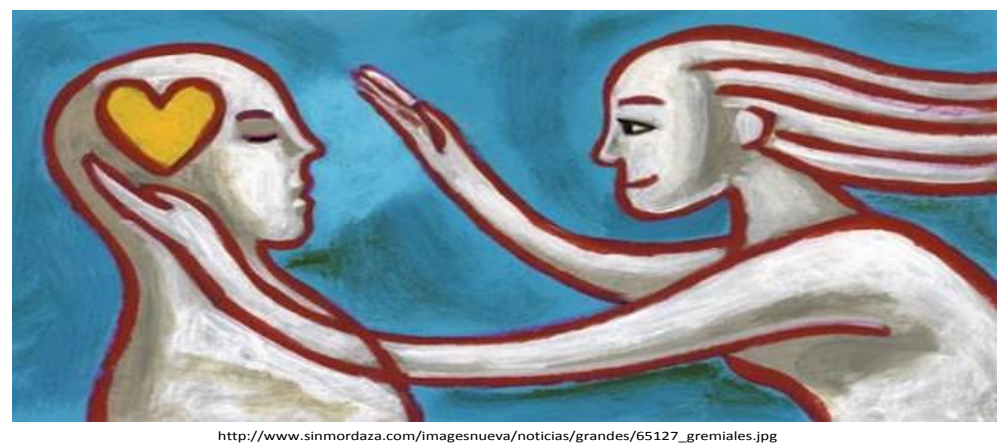

\section{Resumen}

El presente artículo tiene como objetivo mostrar al lector cómo ha sido concebida la Ley 1616 de 2013 en el marco de las experiencias de tres países latinoamericanos (Argentina, Brasil y Chile). En 1990 la OPS/OMS hace una revisión crítica y nota que el proceso de internalización, institucionalización y custodia en psiquiátricos no es descentralizado, participativo, integral, continuo y preventivo. De allí que la salud mental bajo un enfoque comunitario estructure una nueva concepción de los servicios, lo que implica el desarrollo y ejecución de programas para un grupo determinado de personas con el fin de promover, proteger y tratar la salud mental y sus problemas.

Palabras claves: Ley, legislación, salud mental, América Latina.

\section{Summary}

This article aims to show the reader how the law 1616/2013 has been conceived within the framework of the experiences of three Latin American countries (Argentina, Brazil and, Chile). In 1990 PAHO/WHO made a critical review and noted that the process of internalization, institutionalization and, psychiatric custody was not decentralized, nor was it participatory, integrated, continuous and, preventive. Hence, mental health has come up with a new conception of the services under the community approach. This implies the development and implementation of programs for a certain group of people in order to promote, protect and treat mental health and mental health problems.

Keywords: Law, legislation, mental health, Latin America.

${ }^{++\dagger}$ Psicólogo, Esp. Gerencia, Universidad Pontificia Bolivariana y Estudiante Maestría en Salud Mental Comunitaria, Universidad El Bosque, Bogotá-Colombia.

¥¥¥ Psicóloga, Estudiante Maestría en Salud Mental Comunitaria, Universidad El Bosque, Bogotá-Colombia.

§§§ Psicólogo, Esp. Psicología Clínica, U. Católica y Estudiante Maestría en Salud Mental Comunitaria, Universidad El Bosque, Bogotá-Colombia - japedrazap@gmail.com.

**** Psicóloga, Esp. Desarrollo, Universidad del Norte y Estudiante Maestría en Salud Mental Comunitaria, Universidad El Bosque, Bogotá-Colombia. 


\section{A modo de introducción}

El presente artículo tiene como objetivo mostrar al lector cómo ha sido concebida la Ley 1616 de 2013, primera ley en salud mental colombiana y que se encuentra en proceso de reglamentación, en el marco de las experiencias de tres países Latinoamericanos, los cuales, según la Organización Panamericana de la Salud, son abanderados en el tema de salud mental, no solo por su compromiso desde la Declaración de Caracas, para reestructurar su sistema de atención psiquiátrica, sino por sus legislaciones progresistas en materia de derechos humanos y civiles.

\section{Cada país con un enfoque diferente}

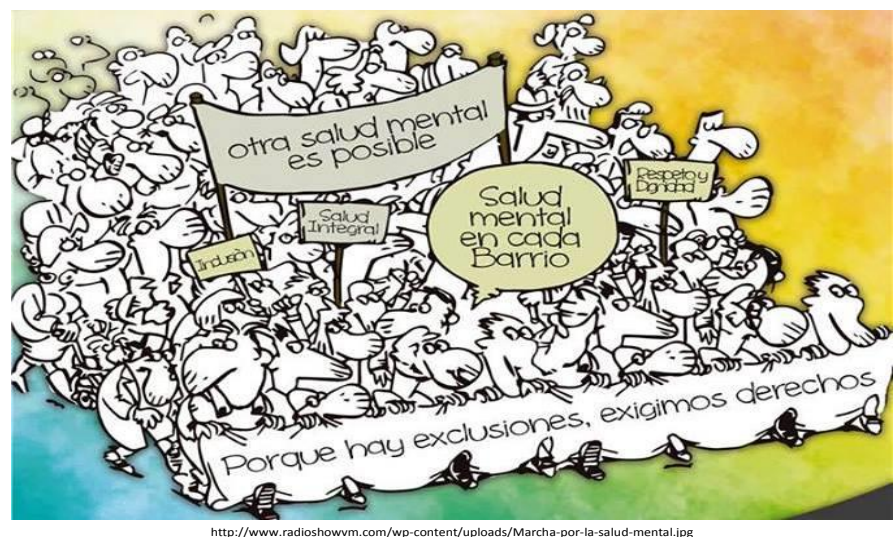

Es importante apuntar que en diferentes legislaciones en políticas públicas en salud mental, tanto de América Latina como del Caribe (AL y C.), como, por ejemplo, en países como Brasil, Argentina, Chile y Colombia, se observan diferencias tanto en los principios y fundamentos de la política, como en los mecanismos planteados para su implementación; de allí que se pueda aseverar que la política pública en salud mental presenta distintos desafíos para su regulación.

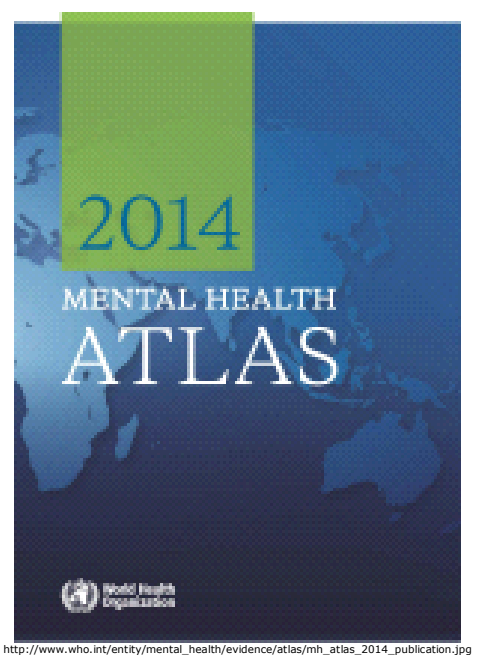

Un esfuerzo de la OMS que se conoce como El Proyecto Atlas de Salud Mental, presenta un panorama desalentador en cuanto al déficit en servicios de salud mental en AL y C.: "los niveles de inversión en salud mental, así como la disponibilidad de camas psiquiátricas, médicos psiquiatras y otros 
profesionales de la Salud Mental en estos países están rezagados si se compara con Estados Unidos y Canadá" (1).

\section{Comunidad: la clave}

Con respecto a la atención en salud mental, la OMS afirma que los sistemas de salud deben enfocar los esfuerzos en lo comunitario, pues lo que se busca privilegiar es una salud mental integrada en la atención general en salud y en otros ámbitos de la vida social como la educación, en definitiva es una apuesta por el trabajo y las actividades que promuevan el bienestar en general (2).

Es importante resaltar que la acción comunitaria en temas de salud en general y de salud mental en específico, es reforzada por el hecho de que los planes en salud mental de muchos países latinoamericanos están cimentados sobre un modelo biopsicosocial. En este escenario las experiencias de países como Argentina, Brasil y Chile se han consolidado a partir de modelos alternativos que han mostrado su efectividad en el abordaje de temas relativos a la salud mental, como es el caso de la implementación de modelos comunitarios, donde, como cita la ley colombiana: "se propone la participación y el compromiso activo de la comunidad, no solo del personal sanitario... sino de los asistentes sociales, la policía, los magistrados, jueces, y demás interesados" (2).

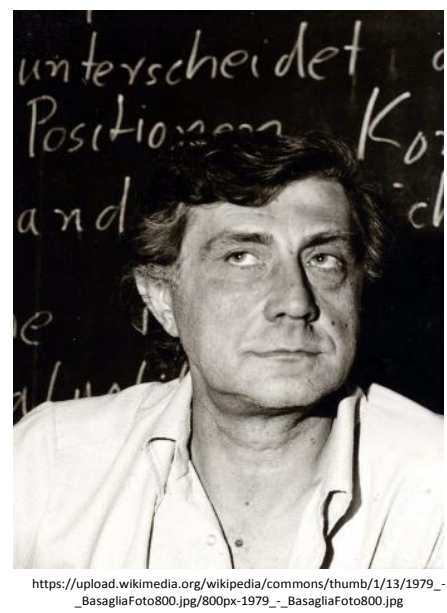

El modelo comunitario se constituye en un cambio de paradigma frente a la atención de personas con trastornos mentales crónicos y "una nueva concepción de la oferta de servicios de salud mental" (3). En esta visión es importante resaltar que la calidad de la atención en salud mental no depende exclusivamente de los recursos disponibles, sino de conceptos como diversidad y calidad, multidisciplinariedad e interdisciplinariedad, aprendizaje, cambio y complejidad (4).

Bajo este paradigma se formulan una serie de indicadores de servicios de salud mental comunitaria que permiten garantizar el tratamiento y el respeto a los derechos de los ciudadanos. Uno de ellos es el modelo de referencia biopsicosocial para afrontar las diferentes caras del sufrimiento psíquico, el cual no puede ser concebido solo desde procesos médicos. Otro indicador será la accesibilidad (apertura 24 horas/día, siete días por semana), sin intermediarios, así puede decirse que "la institución se acerca al ciudadano" (5). 


\section{Un poco de historia...}

En 1990 la OPS/OMS hace una revisión crítica de la "atención psiquiátrica convencional" y encuentra que no es "descentralizada, participativa, integral, continua y preventiva" (6). Nota, además, que los procesos de internalización, institucionalización y custodia en psiquiátricos se presentan como una modalidad única que obstaculizaban el logro de dichos objetivos, que luego se plantearían en la Declaración de Caracas, donde se estipularía que "las legislaciones de los países deben ajustarse de manera que, a) aseguren el respeto de los derechos humanos y civiles de los enfermos mentales y b) promuevan la organización de servicios comunitarios de salud mental que aseguren su cumplimiento" (7).

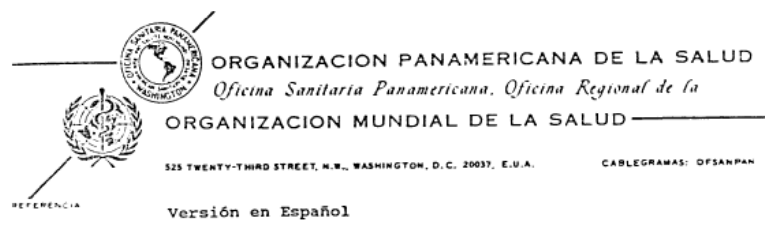

D E C L A R A C I O N

Caracas, Venezuela

14 de noviembre de 1990

Así, la Declaración de Caracas constituye el punto de partida del análisis de las diferentes legislaciones, dado que este documento enfatiza y "promueve un marco legal que establece la protección de los derechos humanos, la vigencia de la democracia en los servicios y, aunque menos explícitamente, la equidad" (8).

En noviembre de 2005, la OPS, la OMS y el gobierno de Brasil citaron a la "Conferencia Regional para la Reforma de los Servicios de Salud Mental", buscando hacer un balance 15 años después de la promulgación de la Declaración de Caracas para analizar los progresos alcanzados en la región en materia de salud mental.

Como mencionan Levav y González, entre las conclusiones de la Conferencia de Brasilia, una de las más importantes a la que se llegó es que "de las experiencias vividas a lo largo de estos años se derivan importantes lecciones, cuya aplicación en el futuro puede ser crucial para la culminación del proceso de reforma" (8). Plantean de igual manera, que es imperativo documentar y difundir las experiencias exitosas en la atención en salud mental de los países de la región, "acción que se verá favorecida por la promoción de programas de cooperación entre países" (8).

De la misma manera, los autores resaltan que el escenario de la Conferencia brindó una oportunidad importante para los países participantes para establecer un marco de referencia, ofrecer las bases de "un programa para la acción y consolidar el flujo tanto de recursos latinoamericanos como de países fuera de la Región que generosamente prestaron apoyo" (8).

Como bien se menciona en el documento de seguimiento a la "Reforma de los servicios de salud mental", no estaba estipulado en la Declaración de Caracas la formulación obligatoria de planes nacionales de salud mental, aun así, "16 países de la Región de las Américas (lo cual representa el $56,5 \%$ del total de ellos) formularon un plan nacional de salud mental entre los años 1991 y 2001 " (8). 


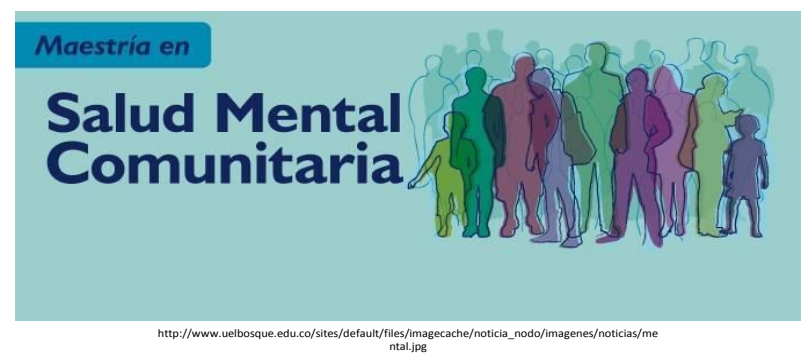

De acuerdo a lo anterior, en lo referente a la voluntad política a favor de la salud mental comunitaria, se pueden ilustrar los hechos ocurridos en tres países latinoamericanos que cuentan con leyes en esta materia. Las normas jurídicas instauradas, por ejemplo, en Río Negro (Argentina) y Brasil forman parte de las leyes promulgadas por el poder legislativo, mientras que, en el caso chileno, dichas normas tienen el respaldo de un documento oficial del "Ministerio de Salud - Plan Nacional de Salud Mental y Psiquiatría" (6).

En Chile, como lo destaca la revisión realizada por Minoletti y Zaccaria, a los 10 años de experiencia exitosa del Plan: "la aplicación de estrategias de salud pública se agrupó en dos planes nacionales de salud mental y psiquiatría puestos en marcha por el Ministerio de Salud en los años 1993 y 2000" (9). De hecho, señalan que "con el primer plan se introdujeron prácticas innovadoras que sirvieron de base para formular el segundo plan, el cual estableció un modelo más elaborado de red, de atención y de programas de actividades que abarcaban los problemas prioritarios" (9).

Brasil, como país firmante de la Declaración de Caracas, se adhiere a ella tras un largo movimiento de trabajadores en salud mental, este hecho quedó ratificado en la ley 9867, del 10 de noviembre de 1999 (10), donde se reconoce la necesidad de incluir y de realizar una integración social de las personas con alguna desventaja, entre ellas las personas con deficiencias psíquicas y con discapacidad mental, que dependen de la asistencia psiquiátrica permanente, y los egresados de los hospitales psiquiátricos.

\section{El caso colombiano}

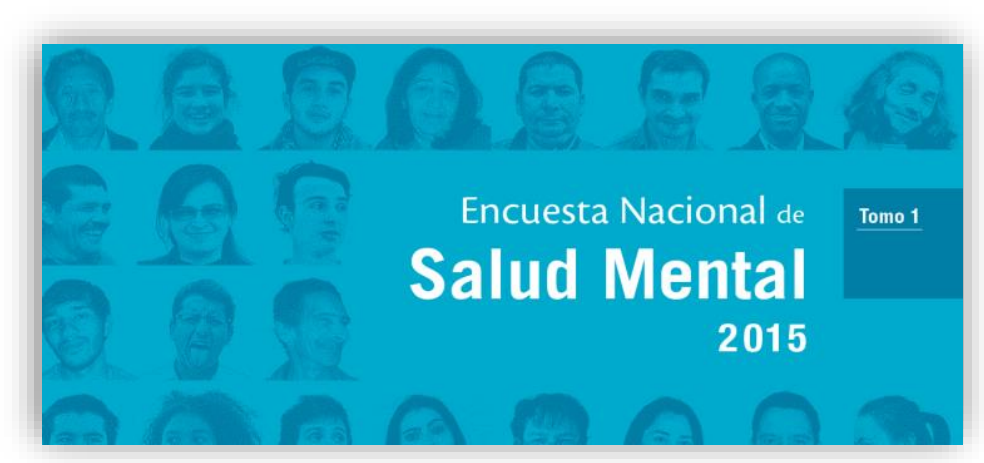

Estudios en Colombia han mostrado un aumento considerable en el número de personas que padecen trastornos mentales que tienen dificultades junto con sus familias para acceder a tratamientos y a servicios de atención calificados. Esta situación se agudiza porque en el país existe una brecha muy amplia entre la formulación de las políticas públicas y la implementación de las mismas; a esto se añade la preeminencia de un modelo biomédico de atención basado en la enfermedad. 
Sumado a lo anterior, la elaboración de una política oficial y su aprobación e implementación constituyen un proceso largo y complejo, lo cual explica por qué, en la región de las Américas, uno de cada cuatro países no cuenta con una política nacional de salud mental y por qué, en aquellos que la han formulado, su implementación aún es incompleta.

Según Desviat "la legislación de salud mental no debe ser vista como un suceso único, sino como un proceso continuo que evoluciona con el tiempo" (5). Esto implica que la legislación debe ser evaluada, revisada y reestructurada conforme se evoluciona en la "atención, tratamiento y rehabilitación de los trastornos mentales", y a partir de los avances en el desarrollo y prestación de los diferentes servicios de salud mental de cada uno de los países (5).

\section{Buscando la integralidad}

Esto podría explicar de manera somera por qué, hasta la promulgación de la ley 1616 del año 2013, el Ministerio de Salud y Protección Social de Colombia no asumía la responsabilidad de brindar una atención integral e integrada en salud mental; es a partir de su expedición que se promueve, "en el marco de la atención primaria en salud, el modelo de atención integral e integrada, los protocolos de atención y las guías de atención integral en salud mental" (6). Sin embargo, en los distintos programas propuestos sigue existiendo una necesidad constante por fomentar una salud social, emocional y mental como una prioridad pública, donde la promoción y la prevención deben organizarse como metas importantes y permanentes en salud pública (10).

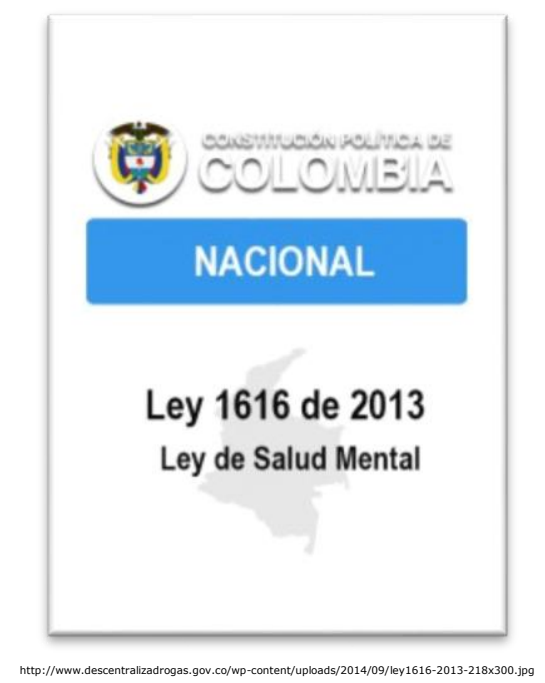

Señala la norma que, para su aplicación, debe existir una "atención integral e integrada en la salud mental", entendida la atención integral como "la concurrencia del talento humano y los recursos suficientes y pertinentes en salud para responder a las necesidades de salud mental de la población, incluyendo la promoción, prevención, diagnóstico precoz, tratamiento, rehabilitación en la salud e inclusión social" (6).

A pesar de este avance en términos legislativos, en lo referente a la postulación e implantación de estas legislaciones en salud mental, Colombia se encuentra en desventaja en tiempo y en restauración de servicios. A la fecha la ley 1616 de 2013 se encuentra en un proceso de reglamentación, pero reconoce la necesidad constante de fomentar una salud social, emocional y mental como una prioridad política (5). 
Como lo menciona la OMS "el propósito fundamental de la legislación en salud mental es el de proteger, promover y mejorar la calidad de vida y el bienestar mental de los ciudadanos. Donde la necesidad de tener una legislación en salud mental surge de la creciente comprensión de la carga personal, social y económica que significan los trastornos mentales en todo el mundo" (11).

\section{A modo de reflexión...}

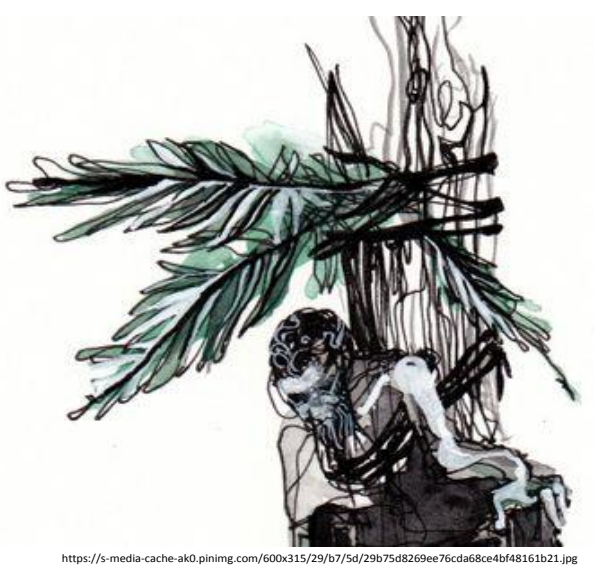

El desconocimiento del fenómeno mental en todas sus vertientes trae consigo una serie de prácticas que se constituyen en violaciones de los derechos humanos, por ejemplo, en algunos países, entre ellos Colombia, "las personas con trastornos mentales son atadas a los árboles en las afueras de sus comunidades, donde se dejan semi-desnudas o con harapos, son golpeados regularmente, y se les restringe el acceso a la comida" (12).

\section{Por ello...}

Este tipo de prácticas y las condiciones de vulnerabilidad, por no decir de abandono, a las que se ven sometidas las personas con trastornos mentales, se constituyen en una de las motivaciones más fuertes para reformar las políticas nacionales en materia de salud mental y la forma en que los sistemas de salud locales, nacionales y regionales prestan dichos servicios en esta materia (13).

En este contexto, la legislación debe ser entendida como una herramienta particularmente poderosa para la promoción de los derechos fundamentales. Bronislaw Malinowski delimita de un modo más claro esta misma idea cuando define claramente la ley como "un conjunto de obligaciones vinculantes sentidas y miradas como una obligación por una de las partes y reconocidas como un derecho por la otra" (14).

Específicamente la legislación en salud mental es "un marco legal que asegura la consideración de temas complejos, como el acceso a los servicios de salud mental, la prestación de una atención con calidad humana y técnica, la rehabilitación y el seguimiento, la total integración de las personas con trastornos mentales a la comunidad y la promoción de la salud mental en diferentes sectores de la sociedad" (15).

De este modo, la salud pública, que se defiende en este artículo, está enmarcada en la salud mental comunitaria, lo que implica el desarrollo y ejecución de programas para un grupo determinado de personas con el fin de promover, proteger y tratar la salud mental y problemas de salud mental (16). 


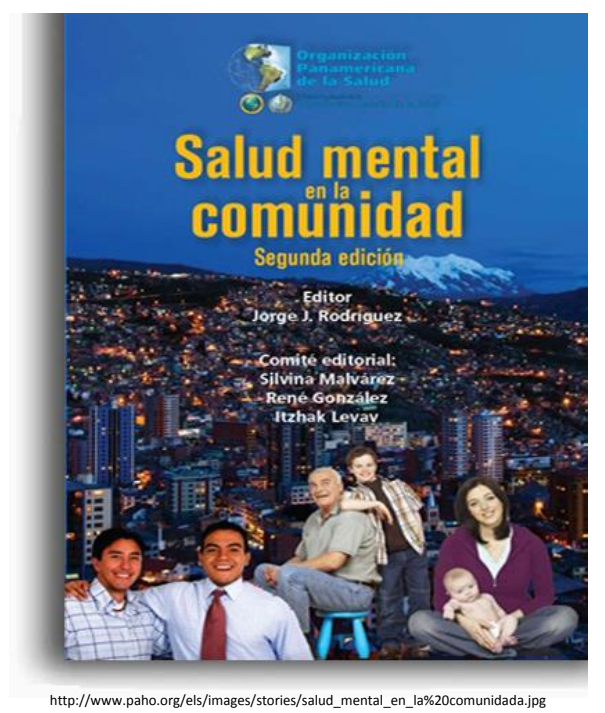

De allí que la salud mental desde este enfoque comunitario estructure una nueva concepción de los servicios, dando el paso de la psiquiatría a los servicios de salud mental y, junto a esto, "tres grandes desplazamientos: del sujeto enfermo a la comunidad, del hospital a los centros comunitarios, y del psiquiatra al equipo" (1). Esta nueva manera de concebir la salud mental representa un modelo radicalmente distinto al predominante, pues en este se entiende al sujeto como representante de un grupo y, por ende, su padecimiento con relación al problema comunitario que representa (17).

En este escenario la formulación de políticas per se constituye un proceso largo y complejo, pues estas hacen manifiesto un "conjunto organizado de valores, principios y objetivos para mejorar la salud mental y reducir la carga de trastornos mentales" (18). Su complejidad está determinada por las interacciones que se dan entre los siguientes elementos: el régimen político, los actores sociales, la cultura social y política y el escenario internacional (19).

\section{Sugerencias...}

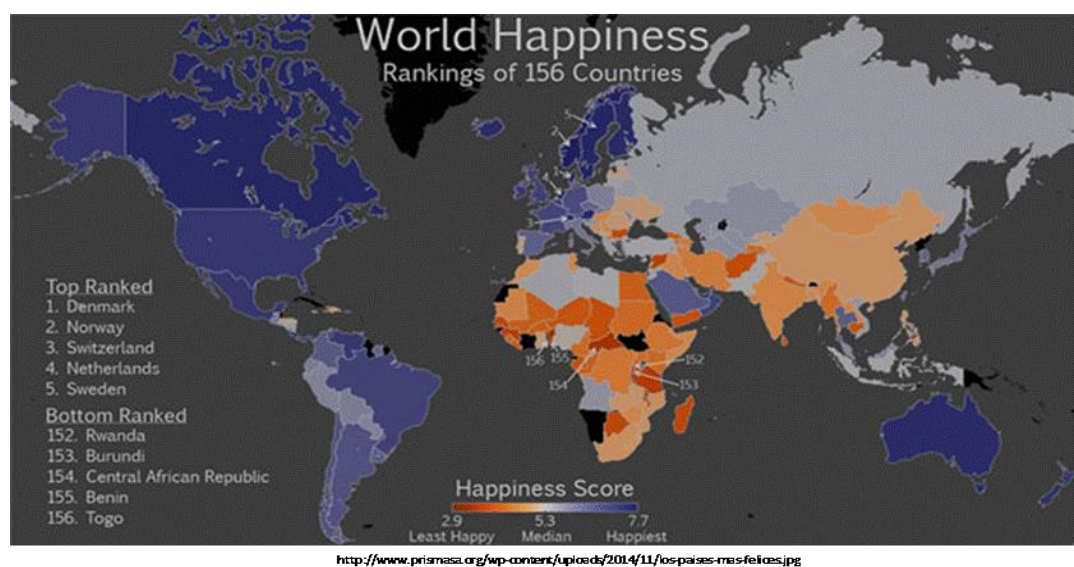

Con base en los argumentos y razones expresados a lo largo del texto y con el objetivo de ampliar el conocimiento de lo que ha ocurrido en los procesos de reforma en atención en salud mental y 
psiquiátrica, se percibe que existe la necesidad de realizar una revisión de las legislaciones de países que han sido considerados como líderes en atención en este campo, esto con el objetivo de comparar en un clima constructivo las políticas en salud mental promulgadas en Colombia con aquellas que se han desarrollado en países que llevan muchos años y pasos adelantados en ese tema.

Existe también la necesidad urgente de un estudio riguroso de las legislaciones de países latinoamericanos que han sido exitosas en sus políticas de promoción de la salud mental y prevención del trastorno mental, pues esto servirá de insumo para la realización de propuestas de atención integral que permitan un abordaje comunitario de la salud mental en el país, ofreciendo los elementos al estado colombiano para que se reglamente y se implemente la legislación actual, además de incentivar la realización de futuras investigaciones en el área de la salud mental comunitaria.

\section{REFERENCIAS}

(1) Rodríguez JJ, Kohn R, Levav I. Epidemiología de los trastornos mentales en América Latina y el Caribe. En: Rodríguez JJ, Malvárez S. González R, Levav I. Salud Mental en la Comunidad. Segunda Edición. Ed. Washington D.C: Organización Panamericana de la Salud: 2009. p 27 - 28.

(2) Ley 1616 /2013 de 21 de enero de Salud Mental (Diario Oficial del Estado, número 48680).

(3) U.S. Department of Health \& HUman Services. Report of the Sugeon General's Conference on Children's Mental Health: A National Action Agenda. Washinton DC: Departament of Healt \& Human Services; 2000.

(4) Larban Vera, J. (2010). El modelo comunitario de atención a la salud mental "continente y contenido". Cuadernos de Psiquiatría y Psicoterapia del Niño y el adolescente.

(5) Desviat M. La Reforma Psiquiátrica 25 años después de la Ley General de Sanidad. Rev Esp Salud Pública. 2011; (85): p. 427-436.

(6) Funk M, Faydi E, Drew N, Minoletti A. Organización de los servicios de salud mental. El modelo comunitario en salud mental. En: Rodríguez JJ, Malvárez S. González R, Levav I. Salud Mental en la Comunidad. Segunda Edición. Ed. Washington D.C: Organización Panamericana de la Salud: 2009. p 105 - 117

(7) OPS, Declaración de Caracas (1990).

(8) Levav, I. \& González, R. (2005) Las raíces de la declaración de Caracas. En: Organización Panamericana de la Salud, La Reforma de los Servicios de Salud Mental: 15 años después de la Declaración de Caracas. Washington, DC: OP.

(9) Minoletti A, Narváez P, Sepúlveda R, Funk M. Desarrollo de políticas y planes de salud mental comunitaria. En: Rodríguez JJ, Malvárez S, González R, Levav I. Salud Mental en la Comunidad. Segunda Edición. Ed. Washington D.C: Organización Panamericana de la Salud; 2009. p. 89-103.

(10) Alberto Fernández Liria MHMBRV. Psicoterapias en el sector público: un marco para la integración. Madrid: Asociación Española de Neuropsiquiatría; 1997.

(11) OMS, Manual de recursos de la OMS sobre salud mental, derechos y legislación (2006).

(12) Hogbonon G. L'Association St Camille. [Internet]. 2005. Disponible en: http://www.aimasm.org/aimacat/stcamille/contingut.html

(13) Funk M, Saraceno B, Drew N, Lund C, Grigg M. Mental health Policy and Plans: Promoting an Optimal Mix of Services in Developing Countries. International Journal of Mental Health. 2004; 33(2): 4-16.

(14) Ochoa C. Derecho Consuetudinario y Pluralismo Jurídico. Cholsamaj 2002. Página 107.

(15) Ley 1616 / 2013 de 21 de enero de Salud Mental. (Diario Oficial del Estado, número 48680).

(16) Asociación Española de Neuropsiquiatría. Principios y objetivos de la salud mental comunitaria. In Desviat M, Moreno A, editors. Acciones de Salud Mental en la Comunidad. Madrid: Exlibris; 2012. p. 21-27

(17) Caplan G. Principios de psiquiatría preventiva Buenos Aires: Paidós; 1966.

(18) Asociación Española de Neuropsiquiatría. Planificación y programación en salud mental. En Desviat M, Moreno A, editors. Acciones de Salud Mental en la Comunidad. Madrid: Exlibris; 2012. p. 104-114.

(19) Alcántara M. Gobernabilidad, crisis y cambios México: Fondo de Cultura Económica; 1995.

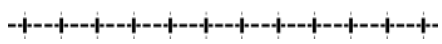

